\title{
Gestión por competencias, modelo empresarial y sus efectos subjetivos. Una mirada desde la Psicología Social Crítica*
}

Competency-Based Work Management, Enterprise Model and its Subjective Effects. A View from Critical Social Psychology

Recibido: marzo 4 de 2013 | Revisado: julio 15 de 2013 | Aceptado: septiembre 15 de 2013

\author{
Patricia Amigot Leache ** \\ LAUREANO MARTÍNEZ \\ Universidad Pública de Navarra, España
}

Doi: 10.11144/Javeriana.UPSY12-4.gcme

Para citar este artículo: Amigot, P. \& Martínez, L. (2013). Gestión por competencias, modelo empresarial y sus efectos subjetivos. Una mirada desde la psicología social crítica. Universitas Psychologica, 12(4), 1073-1084. Doi: 10.11144/Javeriana.UPSY12-4. gcme

Esta investigación fue realizada gracias al apoyo de la Universidad Pública de Navarra, en el marco de actividades del Grupo de Investigación ALTER.

** Departamento de Trabajo Social, Facultad de Ciencias Humanas y Sociales. Campus Arrosadia (31006) Pamplona, Navarra, España. ResearcherID: Martinez, Laureano M-4672-2013; Amigot Leache, Patricia M-4703-2013. E-mails: patricia. amigot@unavarra.es, martinez.laureano@gmail. com

\section{RESUMEN}

Este artículo propone una mirada crítica sobre los discursos de gestión por competencias en el ámbito del trabajo. A partir de una perspectiva psicosocial crítica, se sostiene como hipótesis de lectura que el desarrollo del modelo de las competencias responde a una profunda transformación en el significado del trabajo y de sus formas de gestión y control. Esta transformación se vincula con la implementación de modelos de gestión empresarial en el campo laboral tanto público como privado, lo cual tiene como correlato nuevos modos de subjetivación y de control de la subjetividad de las personas empleadas y desempleadas. El artículo se estructura a partir de los siguientes interrogantes: ¿Qué transformación histórica en el mundo del trabajo permite la emergencia del modelo de las competencias?, ¿Cuál es su especificidad?, ¿Qué mecanismos de control utiliza?, ¿Cuáles son sus efectos subjetivos?

\section{Palabras claves autores}

Gestión por competencias, subjetivación, managemet empresarial, políticas de trabajo.

Palabras clave descriptores

Psicología social crítica, Investigación cualitativa.

\footnotetext{
A B S T R A C T

This article proposes a critical look at the discourses of Competency-based wok management. From the viewpoint of Critical Social Psychology, it is supported as hypothesis that the development of the Competency-based model respond to a deep transformation in the sense of work and its forms of management and control. This transformation entails new modes of subjectivation and control of workers subjectivity. The article is organized around the following questions: What transformations allow the emergence of the Competency-based model? What are its specific characteristics? What are its subjective effects? What mechanisms of control does it use? Key words authors

Competency-based management, subjectivation, enterprise management, labour policies.

Key words plus

KCritical Social Psychology, Qualitative Research.
} 


\section{Introducción}

El objetivo de este artículo es establecer una mirada crítica sobre los efectos subjetivos de los discursos y prácticas vinculados a la gestión por competencias en el mundo del trabajo. Se propone que es posible analizar esos mecanismos en el marco de un conjunto de transformaciones más amplias, vinculadas a las políticas neoliberales, en su intento de extender las lógicas de mercado y el management empresarial a todos los dominios de la sociedad, tanto públicos como privados (Burchell, 1993). Estas transformaciones han dado lugar a una conformación de lo social según el modelo de la empresa, en la que los sujetos son incitados a devenir empresarios de su propia vida (Aubert \& Gaulejac, 1993; Foucault, 2007).

En ese marco, la psicología social permite ampliar el foco de análisis que habitualmente establecen los modelos convencionales de la psicología del trabajo y de las organizaciones, para considerar esa extensión de las lógicas empresariales del mercado desde una perspectiva psicosocial. Esta perspectiva contempla el rol de los aportes de la psicología convencional como partícipes de dicha extensión, ya que en muchas ocasiones operan con la finalidad de amortiguar el efecto de las nuevas exigencias productivas (Pulido-Martínez, 2007) - aunque su papel en la reorganización y control de flujos de poder en el seno de organizaciones concretas se remonta a su propia emergencia (Caballero, 2001) - y permite explorar los efectos de subjetivación que tienen los dispositivos de gestión.

Los autores comparten la consideración de un cierto abandono del ámbito laboral por parte del campo de la psicología social (Pulido-Martínez, 2004; Walkerdine, 2001) y, por tanto, la necesidad de reflexionar acerca de la organización del trabajo y de los discursos y prácticas laborales que operan marcando pautas de acción en dicho campo. En ese sentido, la dimensión crítica de la perspectiva psicosocial tiene que ver fundamentalmente con su carácter problematizador de ámbitos de la realidad social así como de determinados corpus teóricos hegemónicos. De manera resumida, se pueden resaltar tres consideraciones fundamentales de esta mirada crítica, por un lado: a) la naturaleza histórica de la realidad social, lo que sitúa los marcos socioculturales en procesos históricos determinados (Gergen, 1991); b) la consideración de tales realidades sociales como, al menos parcialmente, efecto de la operatividad de dispositivos complejos de poder, tal como ha analizado la obra de Ibáñez (1997) y c) el énfasis en diluir la dicotomía entre lo individual y lo social (Íñiguez, 2001), lo que implica considerar los procesos de configuración social de las identidades y lo subjetivo ${ }^{1}$.

A partir de esos elementos, en este artículo se parte de la consideración histórica de las formas actuales de organización del trabajo, atravesadas por el discurso del management empresarial, y se pretende atender a los dispositivos de poder prácticos y discursivos que intensifican el recurso a cualidades subjetivas, llamadas competencias, en las prácticas de gestión y organización del trabajo, ya sea en el ámbito privado o público, así como a los efectos de tales dispositivos en la configuración de subjetividades. Se intentará mostrar que el modelo de las competencias, en un origen pensado para aspectos específicos de la gestión de organizaciones productivas y educativas, ocupa en la actualidad el centro de las prácticas y discursos laborales, impulsado por la difusión del management empresarial como producto-generador de la sociedad, lo cual conlleva a formas de subjetividad sustentadas en el modelo de la empresa (Ehrenberg, 1991; Gaulejac, 2005).

Para ello se emplean fundamentalmente dos tipos de referencias. Por un lado, un tipo de literatura de carácter normativo, compuesta por publicaciones que han sido y son de gran referencia en el mundo de la gestión empresarial, aunque también cada vez más en el ámbito de la gestión pública. Tal es el caso de autores como David Dubois, William J. Rothwell, Daniel Goleman, Richard Boyatzis, Martha Alles, Claude Lévy-Leboyer, Edward E. Lawler o Leonard Mertens. No es la intención de

1 Recogemos la descripción de la psicología social crítica como una sensibilidad especial que lleva a la apuesta por la elaboración de teorías que cuestionen las asunciones dominantes de la cultura y que propicien la reconsideración de todo aquello que se presenta como evidente, generando así nuevas alternativas de acción social (Doménech \& Ibáñez, 1998). 
este artículo realizar una síntesis o estado de la cuestión de la literatura sobre gestión por competencias, por cierto abundante y heterogénea, sino tomar sus elementos principales, considerados como componentes de dispositivos de poder, para someterlos a un análisis crítico, en un intento por contribuir al desarrollo de la psicología social crítica orientada al mundo del trabajo. Con ese fin se recurre, por otro lado, a un conjunto de autores que desde la psicología social (Caballero \& Blanco, 2007; Crespo \& Serrano, 2012; Hollway, 1991; Ibáñez \& Iñiguez, 1997; Spink, 2011) y desde disciplinas afines como la psicosociología (Ehrenberg 1991, 1998; Gaulejac, 2005) o la sociología orientada al mundo del trabajo (Périlleux, 2003; Zarifian, 2001) ofrecen herramientas para un abordaje crítico del modelo de las competencias, destacando sus consecuencias en la regulación del trabajo y los efectos que ello conlleva a nivel subjetivo.

El artículo parte del siguiente interrogante general: iqué tipo de sujeto trabajador se promueve a partir del modelo de las competencias?, y se organiza en cuatro apartados. En el primero, se reflexiona sobre distintos posicionamientos de los discursos psicológicos en relación con el mundo del trabajo y se proponen los lineamientos de una perspectiva crítica. En el segundo, se hace referencia a una de las principales transformaciones contemporáneas en torno al trabajo: la emergencia del modelo de las competencias. En el tercero, se mencionan los mecanismos de dicho modelo en la gestión de recursos humanos y se analizan los modos de subjetivación que comportan. Por último, se muestra cómo el modelo de las competencias está presente en diversas políticas de empleo, fundamentalmente en aquellas destinadas a promover el espíritu de empresa o emprendedorismo, destacando también sus efectos subjetivos.

\section{La psicología en el ámbito del trabajo y su crítica}

Con el desarrollo del capitalismo, el ámbito del trabajo se ha visto sometido a distintas modalidades de gestión y control, siendo siempre el objetivo lograr el mayor rendimiento posible del proceso de producción. Desde el management científico a las teorías administrativas contemporáneas, diversas vertientes de la psicología han servido y sirven de sustento a las prácticas que tienen como finalidad garantizar la eficiencia del proceso productivo (Hollway, 1991). Un complejo entramado de discursos de raíz "psi" (Rose, 1999), de fuerte carácter normativo, se dirige al dominio de las percepciones de los individuos y los grupos en el ámbito laboral, así como a aspectos organizativos, categorizando y objetivando esas esferas con la finalidad de desarrollar técnicas de gestión y de control del trabajo. Desde la psicología social, Crespo y Serrano Pascual (2012) han mostrado que esos saberes expertos se caracterizan por una fuerte impronta psicologizante, en un sentido preciso: suponen un núcleo explicativo del sujeto como producto de una mente individual o una interioridad personal, relegando las condiciones sociales a un lugar secundario.

En la actualidad, en un claro ejemplo de esta lógica, el discurso de la inteligencia emocional aplicado a la gestión de recursos humanos (Goleman \& Boyatzis, 2008; Goleman \& Cherniss, 2005), conjuga dos concepciones de lo "psi" de gran alcance social. Trasladando los postulados de las neurociencias al campo de los recursos humanos, alternan una representación de la subjetividad reducida a procesos biológicos, fundamentalmente redes neuronales, con una exaltación de la voluntad personal con tintes de omnipotencia; versiones, ambas, que eliminan la consideración de condicionantes sociohistóricos e institucionales. De allí que esos discursos puedan afirmar, por ejemplo, que “... great leaders are those whose behavior powerfully leverages the system of brain interconnectedness." [...grandes líderes son aquellos cuyas conductas aprovechan poderosamente el sistema de interconexión cerebral]. (Goleman \& Boyatzis, 2008, p. 2).

Si esos usos de determinados discursos psicológicos han tenido como principal finalidad la adecuación de los trabajadores ${ }^{2}$ al proceso de producción, una mirada crítica requiere restituir

2 Con el objeto de agilizar la lectura, se optó por el uso generalizado del término masculino, dando por supuesta la inclusión de ambos géneros. 
las prácticas de gestión del trabajo al juego estratégico del que forman parte. Si las prácticas de gestión laboral que se nutren de discursos psicológicos proponen un análisis de la subjetividad partiendo desde el punto de vista individual y encuentran en ella un conjunto de factores que procuran incitar y encauzar bajo criterios de eficacia y eficiencia productiva, una mirada crítica desde la psicología social permite realizar un análisis donde la dimensión subjetiva no es entendida como un conjunto de atributos o facultades que deben desarrollarse y administrarse, sino como producto de relaciones sociales complejas que trascienden el ámbito inmediato de trabajo y que forman parte de estrategias de poder (Ibáñez \& Iñiguez, 1997). En este artículo, al proponer ese modo de análisis de la dimensión subjetiva en el mundo del trabajo, se hace referencia a dos sentidos correlativos de la subjetivación. Por un lado, por subjetivación del trabajo se entiende el conjunto de mecanismos que inducen a una intensificación del compromiso subjetivo en la actividad laboral, a la puesta en juego de los afectos, los valores y las disposiciones relacionales de los trabajadores, de modo que devienen dimensiones necesarias para el proceso de trabajo (Périlleux, 2003). Por otro lado, esos mecanismos conllevan el establecimiento de prácticas que inducen a los sujetos a transformarse a sí mismos, a asumir activamente la tarea de realizar por sí mismos y sobre sí una serie de acciones requeridas para el funcionamiento de los mecanismos sociales -en este caso vinculados al ámbito del trabajo- pero que desplazan su dominio al terreno personal (Ehrenberg, 1991), conduciendo a lo que Gaulejac $(2005,2011)$ llama la "ideología de la realización de sí mismo".

En lo que sigue, se verá cómo el modelo de las competencias pone en juego las dos formas de subjetivación mencionadas. Si en un principio la difusión de la gestión por competencias puede parecer una simple adecuación de las capacidades requeridas a los trabajadores para las nuevas tecnologías y modelos organizacionales -y así es justificada por sus promotores- es posible establecer como hipótesis de trabajo, en cambio, que responde a una profunda transformación en el significado del trabajo mismo y de sus formas de gestión y control, lo cual tiene como correlato nuevos modos de subjetivación y de control de la subjetividad. Por ello, para abordar críticamente el modelo de las competencias, resultan pertinentes los siguientes interrogantes: ¿qué transformación histórica en el mundo del trabajo permite su emergencia?, ¿Cuál es su especificidad?, ¿Qué mecanismos de control utiliza?, ¿Cuáles son sus efectos subjetivos?

\section{Transformaciones en el mundo del trabajo. Dos modelos: el puesto de trabajo y las competencias}

Uno de los principales problemas por resolver en la organización del trabajo, tanto por parte de los empleadores como de personas empleadas, ha sido la relación y la correspondencia entre dos órdenes de distribución jerarquizados: el de las propiedades y requerimientos de los puestos de trabajo y el de los atributos de los trabajadores (Tanguy, 2001). Si en el seno de la sociedad industrial ese problema giraba en torno a la "calificación" de la mano de obra, las transformaciones en el modo de producción capitalista de las últimas décadas, usualmente llamado capitalismo postfordista (Boyer \& Durand, 1998), han trasladado el eje al problema de las "competencias".

El modelo de las competencias surge en un horizonte de crisis de los modelos de gestión centrados en las premisas del taylorismo y del fordismo (Lichtenberger, 1999). Esos modelos organizativos, jerárquicos y burocratizados, serán paulatinamente abandonados en pos de modelos más flexibles, en los que se valora la polivalencia, la iniciativa y la autonomía de los individuos y los grupos de trabajo. En este sentido, como se verá en lo que sigue, el modelo de las competencias surge como contrapunto del modelo del "puesto de trabajo" (Zarifian, 2001).

Pueden destacarse dos modelos históricos que dan respuesta al vínculo problemático entre los trabajadores y los requerimientos de la organización de la producción: el modelo del puesto de trabajo y el modelo de las competencias (Zarifian, 2001). Estos modelos no son estáticos y la emergencia de cada nuevo modelo integra y modifica al anterior (Oiry, 2005). 
El modelo del puesto de trabajo, al que nos referimos habitualmente cuando hablamos de "calificación" o de "empleo calificado", surgió con el desarrollo de la gran industria, pero recién se impuso con posterioridad a la Segunda Guerra Mundial. Si bien en la actualidad sigue teniendo gran preponderancia, la aparición del modelo de las competencias ha transformado en buena parte sus mecanismos.

La emergencia del primer modelo nos remite a los debates que tuvieron lugar en los orígenes y desarrollo histórico de la industrialización, cuando se construye, de la mano del management científico, el objeto "trabajo" como algo que puede ser objetivado, analizado, racionalizado y cuantificado, independientemente de quién lo realice. En ese marco, el trabajador asalariado es concebido como portador de ciertas capacidades necesarias para realizar determinadas tareas. La "calificación" se refiere a la relación entre el puesto de trabajo y las capacidades del individuo requeridas para ese puesto, estableciendo una jerarquía en función de la complejidad. La importancia de la noción de "puesto de trabajo" radica en que es el elemento a partir del cual se establecen los criterios de clasificación del trabajo, la selección de la mano de obra, su control, evaluación y remuneración (Lichtenberger, 1999; Zarifian, 2001).

El puesto implica una función precisa en el seno de la organización, un tiempo preciso de duración del trabajo y un conjunto detallado de tareas por realizar. Estas tareas son asignadas "externamente" por parte de la dirección de la organización, por lo que su iniciativa personal queda reducida al cumplimiento de la tarea asignada. En efecto, una de las características centrales de la racionalización fordista-taylorista del trabajo fue la estricta separación entre el trabajo de dirección y el de ejecución. Como prueba de esto, en sus memorias, Henry Ford se jactaba de haber logrado "(...) eliminar del trabajo el arte del hombre" (Ehrenberg, 1991, p. 224, traducción de los autores).

El modelo de las competencias surge de la crisis de ese proceso histórico y esa amalgama institucional. A principios de la década de 1970, la crisis de los mecanismos de la sociedad industrial condujo a un conjunto de especialistas en Estados Unidos y Reino Unido a cuestionar los modelos tanto de formación como de gestión de los trabajadores (Horton, 2000). La publicación en 1973 del artículo "Testing for Competence Rather than for Intelligence" en la revista American Psychologist por parte del psicólogo David McClelland, es señalada como el punto de partida del modelo de las competencias (Blanco Prieto, 2007; Tovar Martínez \& Revilla Castro, 2010). Dicho artículo condensa los principales argumentos en favor del análisis de las competencias aplicado a la educación y al trabajo.

En esa misma línea, Edward E. Lawler, uno de los principales referentes de la gestión por competencias a nivel mundial, publica en 1994 un artículo que tendrá gran difusión en el mundo de la gestión y cuyo título hace referencia a las transformaciones introducidas en el mundo del trabajo por el modelo de las competencias: "From Job-Based to CompetencyBased Organizations". Allí se presentan los principales ejes implicados en las reformas organizacionales y de gestión de recursos humanos: nuevos sistemas de salarios, nuevos sistemas de selección, de formación del personal y de planificación de carreras, nuevas formas de organización y evaluación del desempeño, todo ello teniendo como eje las competencias individuales y no ya las tareas del puesto de trabajo ${ }^{3}$.

Las reformas realizadas en la organización de las empresas y la gestión del trabajo en la década de 1990 tuvieron al modelo de las competencias como criterio predilecto. Estas reformas fueron acompañadas por un boom de la literatura managerial, que operó como discurso normativo sobre las transformaciones que debían llevarse a cabo (Fernández Rodríguez, 2007). Pero los nuevos criterios organizacionales no estaban destinados únicamente a la esfera privada, sino que su alcance se extendió hasta el corazón de la administración pública, profundamente reformada bajo la dirección del New Public Management (Horton, 2000; Osborne, 1993).

3 "Despite its historical utility, there is growing evidence that it may be time for many organizations to move away from a focus on jobs and towards a focus on individuals and their competencies. [A pesar de su utilidad histórica, existe una evidencia creciente de que para muchas organizaciones puede ser el momento de alejarse de un enfoque centrado en las tareas de trabajo para ir hacia un enfoque centrado en las personas y sus competencias]. (Lawler, 1994, p. 4). 
A partir de ese momento, el modelo de las competencias experimentó una creciente preponderancia, tanto en medios académicos como empresariales y gubernamentales. Su desarrollo y consolidación van de la mano con el complejo y heterogéneo conjunto de transformaciones al que se suele referir con el término neoliberalismo y que tienen al modelo de la empresa como principal referencia (Aubert \& Gaulejac, 1993; Foucault, 2007). Desde entonces, el discurso y las prácticas del management empresarial devienen el espíritu de la época para extenderse a nivel global, a instancias de las grandes empresas multinacionales, de organismos internacionales como la Organización para la Cooperación y Desarrollo Económico (OCDE) o la Organización Internacional del Trabajo (OIT) (Mertens, 1996; OIT, 1997) y de las grandes consultoras, como Hay Group, pionera en gestión por competencias, Ernst \& Young, KPMG o PricewaterhouseCoopers.

En la actualidad, se ofrecen diversos modelos y definiciones de las competencias, según el enfoque que se adopte -conductista, funcional o constructivista (Mertens, 1996) - y según el dominio donde se aplique. Aun así, es posible afirmar que el fenómeno de las competencias hace referencia al conjunto de conocimientos, capacidades y actitudes comportamentales requeridas individual o colectivamente para el desempeño laboral: iniciativa, imaginación, innovación, autonomía, flexibilidad, responsabilidad, control de sí mismo, trabajo grupal y toma de decisiones, entre otras. Se trata de la manifestación de conductas que suelen resumirse en los siguientes ejes: el saber, competencia técnica, como conjunto de conocimientos de una persona; el saber hacer, competencia metodológica, que es el conjunto de habilidades adquiridas por la experiencia y el aprendizaje y puestas en práctica en la situación de trabajo; el saber ser, competencia personal, referida al tipo de conductas que se deben seguir y el saber estar, competencia participativa, como la capacidad de interacción del trabajador (Alles, 2005; Blanco Prieto, 2007) ${ }^{4}$.

4 Para un cuadro detallado de las clasificaciones de las competencias según los principales autores de recursos humanos, véase Blanco Prieto (2007), fundamentalmente los capítulos 4 y 5.
Más allá de las diferencias, lo que interesa desde el punto de vista psicosocial es qué conjunto de relaciones sociales objetivas ponen en juego estos mecanismos - entendidos, como se ha dicho, como dispositivos de poder-y cuáles son sus efectos subjetivos. En ese sentido, debe destacarse la dimensión específica que se ve afectada por esas transformaciones y que permite la emergencia y desarrollo del modelo de las competencias. Esa dimensión es el vínculo entre el trabajador y la actividad de trabajo. Si, como se ha mencionado, la racionalización de tipo taylorista predominante hasta mediados de la década de 1970 se caracteriza por una separación del trabajador del dominio del proceso de trabajo y por la prescripción de tareas, en el modelo de las competencias, el trabajo es de alguna forma "reabsorbido" por el individuo que lo realiza, en tanto debe asumir de manera autónoma las zonas de indeterminación generadas por el retroceso de la prescripción de tareas (Zarifian, 2001). El trabajador deberá decidir autónomamente qué hacer cuando ya no se le indica cómo realizar una tarea. Los nuevos modelos de gestión de la fuerza de trabajo se ocuparán de restituir el "arte del hombre" que Ford había procurado eliminar. Allí radica la importancia de la autonomía no solo individual sino de los grupos de trabajo, de cuya "sinergia" se espera que surjan decisiones innovadoras. Su complemento es la "iniciativa", en cuanto manifiesta el compromiso del trabajador de afrontar activamente los imprevistos que surgen en una situación de trabajo, a la vez que requiere que se le otorgue confianza desde la organización (Zarifian, 2001). De allí que el intrapreneur o intraemprendedor sea el nuevo perfil de trabajador buscado por las empresas. Es decir, aquella persona que dentro de una organización se conduce con iniciativa, autonomía y asume riesgos en busca de nuevos negocios (Prado, Machado, Mafra \& Campos, 2012). Una suerte de sujeto-empresa dentro de la propia empresa.

\section{La gestión por competencias, sus mecanismos y sus efectos subjetivos}

Desde una perspectiva psicosocial crítica, las formas de gestión del trabajo pueden ser analizadas 
como elementos componentes de una estrategia global de poder, siendo la subjetivación de los trabajadores uno de sus principales efectos. Pero una aclaración debe ser precisada. El mundo del trabajo, como es entendido en la modernidad, siempre ha tenido una fuerte impronta subjetiva. Desde los inicios del capitalismo, la racionalización del trabajo y sus mecanismos disciplinarios tenían como objetivo la moralización de los individuos, esto es, inculcar hábitos de ahorro, fijación de la población a su lugar de trabajo, control de las pasiones y los impulsos violentos, de modo de crear ciudadanos observadores de las leyes, etc. Las relaciones de poder no eran simplemente de sometimiento, sino de producción e incitación de una subjetividad dócil (Foucault, 1975). Pero allí donde la organización científica del trabajo de la era industrial establecía una distancia relativa entre el trabajador y la actividad de trabajo, el modelo de las competencias borra esa distancia y requiere del individuo implicación subjetiva y activa en el trabajo, por lo que puede hablarse de una colonización de la dimensión subjetiva con sus recursos de autonomía e iniciativa, emocionales y cognitivos. El modelo de las competencias, por tanto, comporta una fuerte subjetivación del trabajo, en el sentido expresado inicialmente.

Los críticos del capitalismo industrial señalaron la erosión de la experiencia subjetiva en un universo caracterizado por el trabajo alienante que obstruía los poderes de la vida, la dimensión creativa y expresiva de los seres humanos (Boltanski \& Chiapello, 1999). En el modelo de las competencias, el trabajo tiende a ser "reabsorbido" por el trabajador, es decir, deviene expresión directa de la competencia puesta en práctica por el individuo que trabaja. Una suerte de retorno de la actividad de trabajo sobre el individuo que la efectúa (Zarifian, 2001). Mediante este complejo y paradójico retorno, la experiencia subjetiva vuelve al centro de la actividad productiva, pero esto no conlleva una "liberación", sino nuevas formas de control y de producción de cierta clase de experiencias y, por tanto, de subjetividades específicas. Eso sí, receptoras de promesas de creatividad, autonomía, cooperación e intensificación vital, argumentos seductores característicos de la ideología de la gestión (Boltanski \& Chiapello, 1999; Perilleux, 2003).

El modelo de las competencias se ocupa de la selección, la formación y la evaluación de los trabajadores. Para ello, los discursos propios de ese modelo ofrecen una serie de herramientas con el objetivo de poder reconocer las competencias en un individuo o grupo, fomentarlas y evaluarlas ${ }^{5}$. Estos mecanismos parten de la objetivación de las competencias para traducirlas en indicadores (Tallard, 2001). Si originariamente esos indicadores se ofrecen como un medio para reflejar las competencias de un trabajador o el desempeño productivo de una organización, una vez establecidos tienden a transformar la acción de la organización, puesto que esos indicadores pasan a ser los criterios a partir de los cuales se organizan las actividades. Es decir, esos mecanismos, lejos de ser una simple herramienta de medición y valoración, tienden a adoptar un carácter normativo y performativo, en cuanto terminan por definir el tipo de actividades por efectuar (Dujarier, 2010; Martuccelli, 2010), transformando el sentido de la acción ${ }^{6}$ e incluso sustrayéndolo (Gaulejac, 2005).

Desde el punto de vista psicosocial, estos mecanismos tienen un doble efecto. Por un lado, una fuerte personalización de la actividad productiva, es decir, al movilizar cualidades personales, el trabajo deviene tendencialmente una actividad producto de "uno mismo". Por otro lado, a la personalización se suma la responsabilización individual, como modo de ajustar a ese dominio específico las exigencias del trabajo, de modo que "uno mismo" asuma las actividades como un deber propio, aun cuando se forme parte de un "equipo" de trabajo. Todo

5 Los más destacados son: la "observación” (Lévy-Leboyer, 1997), el "Feedback 360" (Lévy-Leboyer, 2000), la Técnica REP y las Pruebas psicométricas (Blanco Prieto, 2007) y numerosos tipos de entrevistas (Dubois \& Rothwell, 2004; Pereda Marín \& Berrocal, 2011).

6 La evaluación, fundamentalmente mediante indicadores cuantitativos, opera diluyendo el valor de la experiencia cualificada -la experiencia laboral-y reorientando las prácticas hacia la consecución de tales indicadores, lo que produce efectos perversos en el sentido que adquieren las prácticas, sobre todo en el ámbito del empleo en sectores sociales y servicios cuyo rendimiento o valor se dejan difícilmente atrapar en una cuantificación de resultados. (Bureau, 2010).

7 Sobre la utilización de metáforas deportivas en el discurso mana- 
ello bajo el ideal normativo de la "realización de sí mismo" (Gaulejac, 2011), bajo una búsqueda de congruencia entre las aspiraciones personales y los objetivos de la organización.

Por tanto, puede considerarse que las técnicas de selección y evaluación por competencias son productoras de subjetividad en dos niveles: a) por una parte, porque suponen llevar a cabo acciones cuyas normas reguladoras son interiorizadas de manera pragmática y procedimental, es decir, suponen un aprendizaje tácito de formas de hacer que tiende a repetirse y naturalizarse; b) por otra, porque la anticipación permanente de los efectos de la evaluación y reevaluación, intensifica la autovigilancia y el compromiso con criterios de hacer y ser, mediante los que un sujeto va adecuándose a los contextos y objetivos institucionales u organizacionales.

Tal como los autores han expuesto en otro lugar (Amigot \& Martínez, 2013), las técnicas de evaluación pueden interpretarse como habilitadoras de procesos de auto-reificación (Honneth, 2007). Esto es, tanto la intensificación de lo subjetivo puesta en juego en el trabajo como las técnicas dirigidas a controlarla y evaluarla en función de los logros, mueven a los sujetos a producirse a sí mismos en los términos requeridos y valorados. Hay, por tanto, una instrumentalización de las propias cualidades subjetivas sujeta a un permanente control que acentúa el carácter provisional de lo conseguido, así como la exigencia de una permanente flexibilidad.

Como señala Ehrenberg (1998), las representaciones sociales se han desplazado desde un paradigma del "conflicto" a uno de la "carencia" - de fuerza, de energía, de motivación-, lo que se traduce en la demanda de una movilización permanente y autoinducida sujeta a los requerimientos laborales; un esfuerzo puesto a disposición de los objetivos de las organizaciones cuyo correlato es una precarización subjetiva. Así mismo, esta carencia y responsabilización individual implican la fragmentación de la experiencia colectiva, lo que genera dinámicas individualizadoras que operan desdibujando la lógica de los derechos -vinculada al puesto de trabajo y que

gerial, véase Ehrenberg, A. (1991) Le culte de la performance. Paris: Calmann-Lévy. permitían, por ejemplo, la negociación colectiva-, sustituyéndola por una lógica del merecimiento en función de las competencias individuales.

\section{La realización de uno mismo y el emprendizaje como ideal normativo}

El alcance del modelo de las competencias recorre transversalmente el mercado de trabajo tanto en la situación de empleo como la de desempleo o de autoempleo, y la figura de un individuo emprendedor y responsable de la movilización de sus competencias se ha establecido como un eje del imaginario social instituido. Es decir, si en el desempeño de las tareas de la persona empleada se supone el deber de movilizar sus competencias personales, a partir de cuyo resultado será evaluada, su correlato en la situación de desempleo es una responsabilización personal del incremento o adecuación de sus competencias, con la finalidad de encontrar un nuevo empleo o de generar el propio. Las políticas de "mejoras de empleabilidad", de incremento del "capital humano" y de fomento del emprendedorismo dan cuenta de ello.

El análisis de un conjunto de documentos ${ }^{8}$ emitidos por organismos internacionales con la finalidad de establecer los lineamientos de políticas públicas de promoción del emprendedorismo, muestra el estrecho vínculo entre esa figura y el modelo de las competencias. En ellos, se propone que con la finalidad de sostener altos niveles de empleo, deben ser fomentadas "competencias y aptitudes esenciales, tales como la creatividad, el espíritu de iniciativa, la tenacidad, el trabajo en equipo, la comprensión de los riesgos y el sentido de la responsabilidad. Es ese estado del espíritu el que permite a los emprendedores transformar las ideas en acciones y que incrementa además fuertemente su empleabilidad" (Comisión Europea, 2013).

Este tipo de políticas responde a la transformación del mercado de trabajo en un marco de crisis de la "sociedad salarial" (Alonso, 2007; Castel,

8 Sobre las políticas públicas de promoción del "espíritu empresarial" o "emprendedorismo" y competencias, véase para el caso Europeo (Comisión Europea, 2013), para el caso latinoamericano en perspectiva comparada (Kantis, 2004). 
1995), pero su impacto va mucho más allá, puesto que establece una racionalidad que ha modificado el sentido mismo del concepto de empresa. Este ya no designa solo una forma de institución económica, sino una forma de conducta social, una forma de comportamiento dentro del campo social, al punto de que cada individuo es incitado a devenir un empresario de sí mismo (Foucault, 2007; Gaulejac, 2005). Además, en esos documentos, la figura del emprendedor pretende tener vocación universal, en una suerte de intento de desplazamiento del obrero asalariado hacia el emprendedor. De hecho, esta política se dirige a: "los jóvenes, las mujeres, las personas mayores, los inmigrantes y los desempleados" (Comisión Europea, 2013). La conducta empresarial deja de ser una función exclusiva de determinado sector y agente económico, para desplazarse hacia el conjunto de los individuos. Emprender simboliza una creación personal, una aventura posible de ser emprendida por todos (Ehrenberg, 1991).

De este modo, la figura del emprendedor, sustentada en el desarrollo de las competencias de cada individuo, implica un particular vínculo del individuo consigo mismo (Ehrenberg, 1998). Plasmada en consignas tales como "conócete a ti mismo", "ser uno mismo", "autocontrol", "motivación personal" o "inteligencia emocional", implica una suerte de conminación que establece en un mismo discurso la conquista de la identidad personal y la del éxito social. En el modelo del emprendedor y del capital humano, el desarrollo autónomo de las competencias supone la conformación de una personalidad que es la condición de un desempeño exitoso en el campo económico y social. Por todo ello, estas figuras ponen de manifiesto el segundo sentido de la subjetivación al que se ha hecho referencia inicialmente. Es decir, pone en juego una serie de prácticas que inducen a los sujetos a transformarse a sí mismos, a asumir activamente la tarea de realizar por sí mismos y sobre sí una serie de acciones requeridas para el funcionamiento de los mecanismos sociales de producción, bajo el ideal normativo de realización de sí mismos.

Esto conlleva numerosas consecuencias. Si por el lado de la regulación social encontramos flexibilización, intensificación y precarización del trabajo, desde el punto de vista psicológico asistimos a la proliferación de patologías como el estrés, la depresión y la angustia, producto del desgaste mental en el trabajo (Dejours, 2009; Ehrenberg, 1998) y a la tendencia a una atribución interna del fracaso social del individuo. Tal como han señalado algunos autores (Crespo \& Serrano Pascual, 2012; Perilleux, 2003), la exigencia subjetiva permanente y la individualización de los procesos tiene efectos patogénicos, pues generará experiencias de sufrimiento privado -difícilmente comunicable bajo otra clave que no sea la del fracaso- y el recurso a sustancias psicotrópicas o drogas de inserción que permiten aliviarlo y sostener un esfuerzo en la competición, manteniendo la integración social y relacional. Un ideal que por su dinámica competitiva diluye lazos colectivos a pesar de sus llamadas a la cooperación y que invisibiliza los condicionantes socioeconómicos que subyacen a los requerimientos individuales.

En suma, si el modelo de las competencias hace del intraemprendedor el ideal de trabajador en las organizaciones, esa misma lógica se desplaza a la totalidad del mercado de trabajo donde el emprendedor se presenta como el modelo a seguir, en lo que Boutillier (2005) llama el paso de una sociedad salarial a una sociedad de emprendedores, donde los mecanismos y discursos del management empresarial pretenden dar forma a la totalidad de lo social.

\section{Conclusión}

Tal como se ha mostrado, el prisma de la psicología social crítica, al restituir un mecanismo específico como el modelo de las competencias al juego estratégico de poder del que forma parte, permite problematizar efectos no explícitos.

El avance tecnológico del capitalismo y la crisis de los marcos regulatorios de la ciudadanía social marcan una pendiente en la que los riesgos tienden fuertemente a individualizarse. Las fuerzas vitales de los seres humanos son puestas en el centro del proceso productivo, bajo mecanismos de control que ya no se sirven en primer lugar de la coerción externa, sino de una fuerte subjetivación de las pautas de trabajo. La cre- 
ciente fragmentación que esto conlleva dificulta encontrar las respuestas colectivas que antaño emergían en los espacios del trabajo asalariado. La tarea de una reflexión crítica, a la que este artículo procura contribuir, es la de problematizar los mecanismos de poder que modulan las pautas sociales y pretenden establecer ideales normativos hegemónicos. Esta crítica, lejos de ser una tarea unidireccional y definitiva, debe convocar aquellos discursos y prácticas que converjan en la interminable tarea de reflexionar y producir los vínculos que las personas establecen entre ellas y consigo mismas, para intentar buscar respuestas colectivas a los problemas del presente.

La crítica implica una práctica epistémica cuya dimensión política se reconoce de manera explícita y cuyo efecto de fractura en los discursos hegemónicos es ya una respuesta. No obstante, se quiere concluir el artículo mencionando algunas líneas propositivas que pueden resultar interesantes para profundizar en el efecto transformador de la crítica. En este sentido, si, tal como se ha apuntado, los sujetos se ven atrapados en esa paradoja que consiste en tener -y querer- autorrealizarse, esforzarse por auto-producirse como competentes gestionando su propia subjetividad, pero con la exigencia implícita de hacerlo trasmutando en propios los objetivos de la organización -objetivos que además son variables y responden a lógicas macroeconómicas desprovistas de cualquier atención al lazo social y que exceden cualquier propósito individual-, es imprescindible mostrar tal paradoja de una manera similar a la que permite salir de una relación de doble-vínculo: encontrar un lugar de exterioridad que amplíe el análisis y permita comprender este proceso. En palabras de Gaulejac, situarse en un "registro simbólico que permite refundar el sentido fuera del sistema de legitimación interna que todo poder hace funcionar" (2005, p. 311, traducción de los autores). De esta forma, es fundamental restituir un análisis psicosocial que des-individualice la comprensión de los procesos laborales y que des-naturalice el imaginario empresarial. A su vez, esto incita a una elaboración colectiva de sentido que intente captar las resistencias, los malestares e incluso el sinsentido que resultan de las prácticas y discursos normativos -aunque sean idealizadoresneoliberales.

Por tanto, las diversas líneas de acción crítica tienen que ver con la resistencia a la lógica exclusiva del beneficio como criterio para pensar y organizar los marcos sociolaborales y a la desregularización ${ }^{9}$ de los mismos, y con la apuesta por prácticas económicas solidarias, la exigencia de responsabilidad política a las organizaciones y todo aquello que permita re-crear lazos sociales. Cuando la "utilidad" es situada en el lugar del sentido de la actividad humana, lo que se produce es sinsentido y erosión del lazo social en términos de Mauss (Gaulejac, 2005), quien comprendió el don como elemento fundante de la socialidad humana. Probablemente, toda práctica social que integre algo de esta lógica del don permitirá crear condiciones menos instrumentalizadoras y patógenas en términos subjetivos.

Por último, aunque se ha focalizado en una noción de trabajo vinculada con el empleo formal, se considera que una atención más amplia a la actividad económica y al trabajo como actividad humana y social que no esté tan marcada por los procesos y estructuras del mercado occidental, también permitiría reconocer la capacidad creativa de prácticas no formales que componen los mecanismos de sustento (Spink, 2011) de importante parte de la población en muchos lugares del planeta, además de contemplar de manera más profunda el trabajo de cuidados y doméstico, y comprender así de manera simultánea los procesos de opresión globales junto con el reconocimiento de la capacidad renovada y creativa que excede y escapa a los imaginarios neoliberales en su práctica, mostrando maneras complejas y resistentes de dotar colectivamente de sentido al trabajo.

\section{References}

Alles, M. (2005). Desarrollo del talento humano basado en competencias. Buenos Aires: Granica.

9 O a la apuesta por fórmulas que no subordinen los derechos de ciudadanía al empleo formal, como la demanda de garantías de ingreso básico o renta básica (Pateman, 2004). 
Alonso, L. E. (2007). La Crisis de la ciudadanía laboral. Barcelona: Anthropos.

Amigot Leache, P. \& Martínez, L. (2013). Gubernamentalidad neoliberal, subjetividad y transformación de la universidad. La evaluación del profesorado como técnica de normalización. Athenea Digital: Revista de Pensamiento e Investigación Social, 13(1), 99-120.

Aubert, N. \& de Gaulejac, V. (1993). El coste de la excelencia. ¿Del caos a la lógica al caos? Barcelona: Paidós.

Blanco Prieto, A. (2007). Trabajadores competentes. Introducción y reflexiones sobre la gestión de recursos humanos por competencias. Madrid: ESIC.

Boltanski, L. \& Chiapello, E. (1999). El nuevo espíritu del capitalismo. Barcelona: Akal.

Boutillier, S. (2005). De la société salariale à la société entrepreneuriale, valeur travail et capital social. En S. Boutillier \& D. Uzunidis (Eds.), Travailler au XXIe siècle. Nouveaux modes d'organisation du travail (pp. 79-107). Bruxelles: Editions De Boeck Supérieur.

Boyer, R. \& Durand, J. -P. (1998). L'Après-fordisme. París: Syros.

Burchell, G. (1993). Liberal government and techniques of the self. Economy $\mathcal{E}$ Society, 22(3), 266-282.

Bureau, M. -C. (2010). Du travail à la action publique: quand les dispositifs d'évaluation prennent le pouvoir. Cahiers Internationaux de Sociologie, 1(128-129), 161-175.

Caballero Muñoz, D. (2001). Apuntes para una metateoría en psicología social del trabajo. Psicothema, 13(4), 691-699.

Caballero Muñoz, D. \& Blanco Prieto, A. (2007). Competencias para la flexibilidad: la gestión emocional de las organizaciones. Psicothema, 19(4), 616-620.

Castel, R. (1995). Les métamorphoses de la question sociale: une chronique du salariat. París: Fayard.

Comisión Europea. (2013). Plan d'action «Entrepreneuriat 2020». Raviver l'esprit d'entreprise en Europe. Bruselas: Autor. Recuperado de http:// eur-lex.europa.eu/LexUriServ/LexUriServ. do?uri=COM:2012:0795:FIN:fr:PDF

Crespo, E. \& Serrano Pascual, M. A. (2012). La psicologización del trabajo: la desregulación del trabajo y el gobierno de las voluntades. Teoría y Crítica de la Psicología, 2, 33-48.
Dejours, P. (2009). El desgaste mental. Madrid: Modus Laborandi.

Domènech, M. \& Ibáñez, T. (1998). La psicología social como crítica: percepción intelectual del tema. Revista Anthropos: Huellas del Conocimiento, 177, 12-21.

Dubois, D. \& Rothwell, W. J. (2004). Competency-based human resource management. Palo Alto, CA: Davies Back.

Dujarier, M. A. (2010). L'automatisation du jugement sur le travail. Mesurer n'est pas évaluer. Cahiers Internationaux de Sociologie, 128-129, 135-159. doi: 10.3917/cis.128.0135

Ehrenberg, A. (1991). Le culte de la performance. París: Calmann-Lévy.

Ehrenberg, A. (1998). La fatigue d'être soi. Dépression et société. París: Odile Jacob.

Fernández Rodríguez, C. (2007). El discurso del management: tiempo y narración. Madrid: CISS.

Foucault, M. (1975). Surveiller et punir. Naissance de la prison. París: Gallimard.

Foucault, M. (2007). Nacimiento de la biopolítica. Buenos Aires: Fondo de Cultura Económica.

Gaulejac, V., de (2005). La société malade de la gestion. Idéologie gestionnaire, pouvoir managérial et harcèlement social. París: Seuil.

Gaulejac, V., de (2011). L'injonction d'être sujet dans la société hypermoderne : la psychanalyse et l'idéologie de la réalisation de soi-même. Revue Française de Psychanalyse, 75(4), 995-1006.

Gergen, K. J. (1991). El yo saturado. Dilemas de identidad en el mundo contemporáneo. Barcelona: Paidós.

Goleman, D. \& Boyatzis, R. (2008). Social intelligence and the biology of leadership. Harvard Business Review, 86(9), 71-78.

Goleman, D. \& Cherniss, C. (2005). Inteligencia emocional en el trabajo: cómo seleccionar y mejorar la inteligencia emocional en individuos, grupos y organizaciones. Barcelona: Kairós.

Hollway, W. (1991). Work psychology and organizational behaviour: Managing the individual at work. London: Sage.

Honneth, A. (2007). Reificación: un estudio en la teoría del reconocimiento. Buenos Aires: Katz.

Horton, S. (2000). The competency movement: Its origins and impact on the public sector [Intro- 
duction]. The International Journal of Public Sector Management, 13(4), 306-318.

Ibáñez, T. (1997). Why a critical social psychology? En T. Ibáñez \& L. Iñiguez (Eds.), Critical social psychology (pp. 27-41). London: Sage.

Ibáñez, T. \& Íñiguez, L. (Eds.). (1997). Critical social psychology. London: Sage.

Íniguez, L. (2001). Identidad: de lo personal a lo social. Un recorrido conceptual. En E. Crespo \& C. Soldevilla (Eds.), La constitución social de la subjetividad (pp. 209-226). Madrid: Ediciones de la Catarata.

Kantis, H. (2004). Desarrollo emprendedor: América Latina y la experiencia internacional. New York: Banco Interamericano de Desarrollo.

Lawler III, E. E. (1994). "From Job-Based to Competence-Based Organizations”. Journal of Organizational Behavior, Vol. 15, 1, 3-15.

Lévy-Leboyer, C. (1997). Gestión de competencias. Barcelona: Gestión.

Lévy-Leboyer, C. (2000). Feedback 360ํ․ Barcelona: Gestión.

Lichtenberger, Y. (1999). Compétence, organisation du travail et confrontation sociale. Formation Emploi, 67, 93-106.

Martuccelli, D. (2010). Critique de la philosophie de l'évaluation. Cahiers Internationaux de Sociologie, 1(128-129), 27-52. doi: 10.3917/cis.128.0027

McClelland, D. C. (1973). Testing for competence rather than for intelligence. American Psychologist, 28(1), 1-14.

Mertens, L. (1996). Competencia laboral: sistemas, surgimiento y modelos. Montevideo: Cinterfor.

Oiry, E. (2005). Qualification et compétence: deux soeurs jumelles? Revue Française de Gestion, 31(158), 13-34. doi: 10.3166/rfg.158.13-34

Organización Internacional del Trabajo. (1997). Seminario Internacional sobre Formación Basada en Competencia Laboral: Situación Actual y Perspectivas. Montevideo: Cinterfor.

Osborne, P. (1993). Reinventing government: How the entrepreneurial spirit is transforming the public sector. New York: Plume.

Pateman, C. (2004). Democratizing citizenship: Some advantages of a Basic Income Grant. Politics and Society, 32(1), 89-105.
Pereda Marín, S. \& Berrocal, F. (2011). Dirección y gestión de recursos humanos por competencias. Madrid: Editorial Universitaria Ramón Areces.

Périlleux, T. (2003). La subjectivation du travail. Déviance et Société, 27(3), 243-255. doi : 10.3917/ ds. 273.0243

Prado, M. L., Machado, E., Mafra, E. M. \& Campos, F. M. (2012). Intrapreneur: An emerging setting in business economy. IEEE Latin America Transactions, 10(1), 1195-1200.

Pulido-Martínez, C. (2004). En búsqueda de una psicología crítica en los ámbitos laborales. Universitas Psicológica, 3(2), 213-222.

Pulido-Martínez, C. (2007). Produciendo trabajadores modernos: conocimiento psicológico y el mundo del trabajo en el sur. Universitas Psicológica, 6(1), 27-37.

Rose, N. (1999). Governing the soul. The shaping of the private self (2nd ed.). London: Free Association Books.

Spink, P. (2011). ¿Qué pasó con el trabajo? De la centralidad de los zapatos, barcos y lacre a los problemas planteados por los cerdos volando. Athenea Digital, 11(3), 3-24.

Tallard, M. (2001). Lintroduction de la notion de compétence dans les grilles de classification. Genèse et évolution. Sociétés Contemporaines, 41-42, 159-187.

Tanguy, L. (2001). De la evaluación de los puestos de trabajo a la de las cualidades de los trabajadores. Definiciones y usos de la noción de competencias. En E. De la Garza Toledo \& J. C. Neffa (Comps.), El trabajo del futuro. El futuro del trabajo (pp. 111128). Buenos Aires: CLACSO.

Tovar Martínez, F. J. \& Revilla Castro, J. C. (2010). La supuesta neutralidad de la evaluación por competencias. Revista Internacional de Organizaciones, 5, 109-196.

Walkerdine, V. (2001). Critical psychology. International Journal of Critical Psychology, 1, 3-7.

Zarifian, P. (2001). Le modèle de la compétence. París: Liaisons. 